\title{
PROCESSO DE MORTE ENCEFÁLICA: SIGNIFICADO PARA ENFERMEIROS DE UMA UNIDADE DE TERAPIA INTENSIVA
}

\author{
THE BRAIN DEATH PROCESS: \\ MEANINGS GIVEN BY INTENSIVE \\ CARE UNIT NURSES
}

\section{PROCESO DE MUERTE ENCEFÁLICA: SU SIGNIFICADO PARA ENFERMEROS DE UNA UNIDAD DE TERAPIA INTENSIVA}

\author{
Murilo Pedroso Alves ${ }^{1}$ \\ Franciele da Silva Rodrigues ${ }^{2}$ \\ Kamylla Santos da Cunha ${ }^{1}$ \\ Giovana Dorneles Callegaro Higashi ${ }^{3}$ \\ Eliane Regina Pereira do Nascimento ${ }^{4}$ \\ Alacoque Lorenzini Erdmann 5
}

Como citar este artigo: Alves MP, Rodrigues FS, Cunha KS, Higashi GDC, Nascimento ERP, Erdmann AL. Processo de morte encefálica: significado para enfermeiros de uma unidade de terapia intensiva. Rev baiana enferm. 2019;33:e28033.

Objetivo: compreender como os enfermeiros significam o cuidado prestado ao paciente no processo de morte encefálica em uma Unidade de Terapia Intensiva. Método: pesquisa qualitativa com aporte teórico-metodológico na Teoria Fundamentada nos Dados. A coleta de dados foi realizada em uma Unidade de Terapia Intensiva de um hospital no Sul do Brasil de setembro a novembro de 2014. Participaram do estudo 23 enfermeiros distribuídos em três grupos amostrais. A análise teve início com a codificação aberta, seguindo para a codificação axial e para a codificação seletiva, conforme a Teoria Fundamentada nos Dados. Resultados: emergiu da análise dos dados o fenômeno "Reconhecendo o trabalho multiprofissional como potencializador da doação de órgãos e tecidos para transplantes", sustentado por cinco categorias. Conclusão: o cuidado prestado aos pacientes em processo de morte encefálica é significado pelos enfermeiros como gerador da oportunidade de uma nova vida aos múltiplos receptores.

Descritores: Cuidados de Enfermagem. Morte Encefálica. Enfermeiros. Unidade de Terapia Intensiva.

Objective: to understand how nurses signify the care provided to patients with brain death in the Intensive Care Unit. Method: this was a qualitative study based on grounded theory. Data collection took place in an intensive care unit in a hospital in the South region of Brazil, between September and November 2014. Twenty-three nurses participated

\footnotetext{
Enfermeiro(a). Mestre em Enfermagem. Florianópolis, Santa Catarina, Brasil.murilopedrosoalves@gmail.com

Enfermeira. Florianópolis, Santa Catarina, Brasil.

Enfermeira. Doutora em Enfermagem. Professora Adjunta do Curso de Graduação em Enfermagem da Universidade Federal de Santa Maria. Santa Maria, Rio Grande do Sul, Brasil.

4 Enfermeira. Doutora em Enfermagem. Professora Associada do Departamento de Enfermagem da Universidade Federal de Santa Catarina. Florianópolis, Santa Catarina, Brasil.

Enfermeira. Doutora em Filosofia da Enfermagem. Professora Titular do Departamento de Enfermagem da Universidade Federal de Santa Catarina. Florianópolis, Santa Catarina, Brasil.
} 
in the study, distributed in three sample groups. The first step of data analysis was open coding, followed by axial and selective coding, according to grounded theory. Results: data analysis resulted in the phenomenon "Recognizing multiprofessional work as promoting organ and tissue donation for transplantation", sustained by five categories. Conclusion: the care provided to patients with brain death was signified by the nurses as generating an opportunity for a new life for multiple recipients.

Descriptors: Nursing Care. Brain Death. Nurses. Intensive Care Unit.

Objetivo: comprender el significado que le dan los enfermeros a la atención brindada al paciente en proceso de muerte encefálica en una unidad de terapia intensiva. Método: investigación cualitativa con aporte teóricometodológico de la Teoría Fundamentada en los Datos. Datos recolectados en una unidad de terapia intensiva de hospital del sur de Brasil, de setiembre a noviembre de 2014. Participaron 23 enfermeros, divididos en tres grupos muestrales. El análisis se inició con la codificación abierta, mudando luego a codificación axial y codificación selectiva, conforme la Teoría Fundamentada en los Datos. Resultados: del análisis surgió el fenómeno "Reconociendo el trabajo multiprofesional como impulsor de la donación de órganos y tejidos para trasplantes", respaldado por cinco categorías. Conclusión: la atención brindada a los pacientes en proceso de muerte encefálica recibe de los enfermeros un significado generador de la oportunidad de una nueva vida para los múltiples receptores.

Descriptores: Atención de Enfermería. Muerte Encefálica. Enfermeros. Unidades de Cuidados Intensivos.

\section{Introdução}

A Morte Encefálica (ME), caracterizada pela parada total e irreversível das funções encefálicas de causa conhecida, de modo indiscutível, manifesta-se por coma aperceptivo, com ausência de resposta motora supraespinhal e apneia. Entre as principais causas estão: hemorragia intracraniana, trauma e lesão cerebral isquêmica. Para o diagnóstico, é necessária a realização de exame clínico complementar, que comprove a ausência de atividade elétrica cerebral ou ausência metabólica cerebral, ou ausência de perfusão tecidual cerebral registrado no termo de declaração de ME por dois médicos, não necessariamente neurologistas, em um intervalo de tempo específico para cada faixa etária ${ }^{(1-2)}$.

Segundo dados de 2015 da Associação Brasileira de Transplante de Órgãos, o maior número de diagnósticos de ME ocorre nas Unidades de Terapia Intensiva (UTI). O cuidado no processo de ME é complexo, podendo levar a diversas complicações deletérias para o potencial doador. Sendo assim, os diversos profissionais envolvidos nesse processo, em especial os enfermeiros, precisam estar capacitados para o exercício de condutas importantes no controle hemodinâmico, hídrico e na monitoração dos pacientes. Esses procedimentos são necessários para que a doação dos órgãos ocorra de maneira satisfatória $^{(3-5)}$.

A notificação para possíveis doações de órgãos e tecidos para transplantes vem crescendo mundialmente. Conforme apontam estudos nacionais e internacionais, o Brasil é o país referência, por possuir o maior serviço público de transplantes no mundo; consequentemente é notório seu destaque no contexto mundial de doação de órgãos e tecidos para transplantes. Em 2017 foram notificados 10.629 casos de ME, efetivando 3.415 doações. A região Sul do Brasil destaca-se com a notificação de 2.467 e efetivação de 1.004 doações. Em Santa Catarina, houve um crescimento de doadores de 10,9\% entre 2016 e 2017, efetivando 282 doações em 2017. As doações efetuadas dos últimos cinco anos vêm contribuindo com o crescimento de transplantes de órgãos mais complexos, como coração, pulmão e medula óssea. No período de 2010 a 2017, o Brasil registrou um avanço de 69\% nos números de doadores de órgãos e tecidos, superando padrões internacionais ${ }^{(1,6-7)}$.

Os cuidados realizados no paciente com diagnóstico de ME visam a manutenção do corpo em condições favoráveis para a doação de órgãos e tecidos, por meio de condutas precisas e complexas realizadas pela equipe multiprofissional 
de saúde. Esses procedimentos são importantes para possibilitar a oportunidade de salvar outras vidas que estão à espera de um transplante ${ }^{(6,8-9)}$.

Nesse contexto, o enfermeiro tem como responsabilidade o planejamento, a execução, coordenação, supervisão e avaliação dos procedimentos de enfermagem prestados aos possíveis doadores de órgãos e tecidos ${ }^{(10)}$. Todas as atribuições do enfermeiro nesse cenário objetivam a otimização do processo de doação e captação de órgãos e tecidos para fins de transplantes, e são intrínsecas ao cuidado dos pacientes com diagnóstico de ME, uma vez que é a enfermagem que acompanha esses pacientes constantemente ${ }^{(3,9-10)}$.

Dada a importância da enfermagem nesse contexto e frente à realidade apontada, o presente estudo tem como objetivo compreender como os enfermeiros significam o cuidado prestado ao paciente no processo de ME em uma UTI.

\section{Método}

Estudo qualitativo com aporte teórico-metodológico na Teoria Fundamentada nos Dados (TFD), a qual possibilita a compreensão dos fenômenos sociais na perspectiva dos significados das relações e interações entre os sujeitos ${ }^{(11)}$. O cenário de coleta de dados foi uma UTI em um hospital referência em doação e transplantes de órgãos e tecidos no Sul do Brasil. A coleta de dados ocorreu de setembro a novembro de 2014, por meio de entrevistas abertas e individuais no local de trabalho dos participantes, gravadas com o auxílio de um dispositivo eletrônico de áudio de voz. A amostragem teórica deste estudo foi composta por 23 participantes distribuídos em três grupos amostrais.

O primeiro grupo amostral foi composto por nove enfermeiros que atenderam aos critérios de inclusão: ser enfermeiro intensivista, ambos os sexos, atuando na UTI há pelo menos seis meses, com experiência no cuidado com o paciente em ME. A abertura do diálogo foi guiada pela seguinte questão norteadora: "Fale-me como você significa o cuidado dirigido ao paciente em processo de ME na UTI."
A análise do primeiro grupo amostral possibilitou que os dados indicassem a necessidade da inclusão dos enfermeiros da Comissão Intra-Hospitalar de Doação de Órgãos e Tecidos para Transplantes (CIHDOTT), uma vez que esses profissionais participam do cuidado com o paciente em ME na UTI, além de facilitarem o processo de doação de órgãos e tecidos para transplantes. Desse modo, o segundo grupo amostral foi formado por quatro enfermeiros atuantes da CIHDOTT, que atenderam aos critérios de inclusão: enfermeiros atuantes na CIHDOTT há pelo menos seis meses. A abertura do diálogo ocorreu com a seguinte questão: "Como você, enfermeiro da CIHDOTT, significa e vivencia o processo de cuidar de um paciente em ME?"

Os dados levantados pelos profissionais da CIHDOTT enfatizaram a formação acadêmica do enfermeiro. Assim, foi necessário compreender a importância do processo de formação do enfermeiro na perspectiva do cuidado com o paciente em processo de ME. Nesse sentido, a fim de atingir maior consistência teórica, o terceiro grupo amostral foi constituído por cinco enfermeiros recém-egressos, respeitando, como critério de inclusão, ter vivenciado a ME durante a formação, e cinco docentes do Curso de Graduação em Enfermagem de uma universidade pública, respeitando, como critério de inclusão, experiência em UTI e cuidado com o paciente em ME. Com os recém-egressos, a abertura do diálogo deu-se por meio da seguinte questão norteadora: "Fale sobre a sua experiência no processo de cuidado com o paciente em ME durante a graduação." Com os docentes, utilizou-se a seguinte questão norteadora: "Fale-me sobre a sua experiência no processo de cuidado com o paciente em ME na perspectiva docente."

O critério de exclusão adotado para este estudo foi estar afastado do trabalho, por qualquer motivo, durante o período da coleta de dados. A repetição de informações sobre o fenômeno e a ausência de novos elementos relevantes para a análise e consolidação das categorias e subcategorias em suas propriedades e dimensões tornou possível obter-se a saturação teórica dos dados. O processo de análise teve início com a 
codificação aberta, seguindo para a codificação axial e finalizando com a codificação seletiva, conforme preconiza o método em questão ${ }^{(11)}$.

O software NVIVO ${ }^{\circledR} 10$ foi utilizado para ordenar e organizar os dados no processo de análise e codificação. O processo analítico, sistematizado segundo o modelo paradigmático, foi constituído de cinco componentes (contexto, causa, condição interveniente, estratégia e consequência) que explicam e sustentam o fenômeno.

O desenvolvimento deste estudo atendeu aos preceitos éticos recomendados pela Resolução n. 466/12 do Conselho Nacional de Saúde ${ }^{(12)}$ e foi aprovado pelo Comitê de Ética em Pesquisa da Secretaria Estadual de Saúde (SES) de Santa Catarina sob protocolo n. 2014/0010. Os participantes, esclarecidos sobre o objetivo e a metodologia da pesquisa, assinaram o Termo de Consentimento Livre e Esclarecido. Suas falas foram identificadas por letra do alfabeto - letra E correspondente a enfermeiros; C, a enfermeiros da CIHDOTT; EG, a enfermeiros que vivenciaram a ME durante a graduação; e D, a enfermeiros docentes - seguida do número da entrevista.

\section{Resultados}

A inter-relação das categorias e subcategorias que emergiram dos dados permitiu revelar o fenômeno "Reconhecendo o trabalho multiprofissional como potencializador da doação de órgãos e tecidos para transplantes", representado na Figura 1.

Figura 1 - Representação do fenômeno central por meio do modelo paradigmático: reconhecendo o trabalho multiprofissional como potencializador da doação de órgãos e tecidos para transplantes

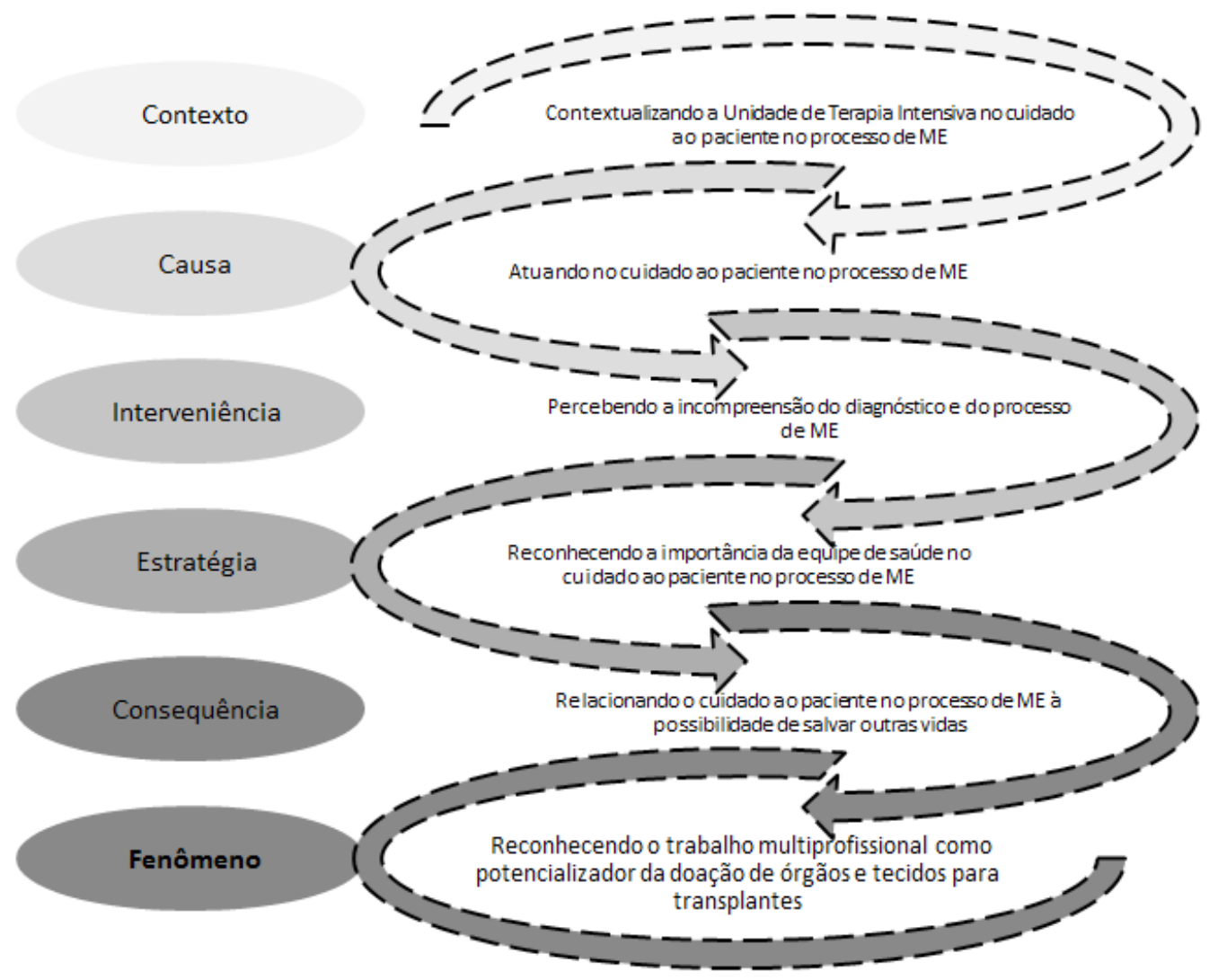

Fonte: Elaboração própria.

A primeira categoria - "Contextualizando a UTI no cuidado com o paciente no processo de ME" - refere-se ao componente contexto. Revela que a UTI, por ser um ambiente permeado por tecnologias, cuidados intensivos e presença contínua de profissionais especializados, é o 
ambiente hospitalar adequado para prestar um cuidado seguro e de qualidade aos pacientes no processo de ME. Isso ocorre principalmente porque esses pacientes precisam de cuidado constante e necessitam de tecnologias específicas e necessárias para o diagnóstico de ME e preservação do corpo para a possível doação de órgãos e tecidos.

A UTI é um ambiente adequado. Tem todo material necessário, tecnologia necessária, pessoal qualificado para lidar com a realidade do paciente em $M E$. Não vejo outro lugar do hospital tão bem adequado para isso, para que preste um cuidado de qualidade. (E7).

Os dados evidenciam que os cuidados prestados pelos profissionais que atuam na UTI aos pacientes no processo de ME são realizados de acordo com as recomendações da Associação de Medicina Intensiva Brasileira (AMIB) e da Associação Brasileira de Enfermagem e Terapia Intensiva (ABENTI), que estão pautadas na legislação brasileira e em condutas baseadas em evidências científicas nacionais e internacionais.

Nossa assistência é pautada pela ciência. Pelas recomendações da $A M I B$ [Associação de Medicina Intensiva Brasileira] $e$ da ABENTI [Associação Brasileira de Enfermagem e Terapia Intensiva], que seguem recomendações internacionais. Não há dúvidas da qualidade do cuidado prestado aqui na UTT. (E2).

Os dados evidenciaram ainda que, por mais específico que seja cuidar de um paciente no processo de ME, a assistência desses pacientes é igualada à dos pacientes com possibilidade terapêutica de cura, principalmente para não discriminar e nem priorizar um ou outro paciente, pois todos, frente à humanização que permeia a assistência de enfermagem, possuem a mesma importância na perspectiva do cuidado.

O cuidado ao paciente em ME é feito de forma tão bumanizada quanto o que é feito ao paciente com possibilidade terapêutica de cura. Não distinguimos isso. O que há distinção é na intensidade do cuidado para manter esses órgãos "vivos". (E9).

A segunda categoria "Atuando no cuidado com o paciente no processo de ME" refere-se ao componente "causa". Revela que os enfermeiros possuem competências técnico-científicas para reconhecer o diagnóstico de $\mathrm{ME}$ e atuar no cuidado direto aos pacientes no processo de ME, junto à equipe multiprofissional, uma vez que possuem formação para o desenvolvimento do raciocínio clínico frente a essa realidade.

\begin{abstract}
E preciso que se desenvolva o raciocínio clínico para perceber os sinais clínicos da ME e pensar clinicamente, comunicar ao médico para que possa dar início ao protocolo. Tudo isso é muito complexo. Podemos levantar hipóteses a partir do raciocínio clínico, contribuir de fato nesse cuidado. (E10).
\end{abstract}

Além disso, os dados apontam que os enfermeiros possuem competências para avaliar não somente a hipótese diagnóstica, como também as condições clínicas dos pacientes no processo de $\mathrm{ME}$, de forma constante, contribuindo efetivamente no cuidado direto ao paciente e consequentemente no processo de doação de órgãos e tecidos para transplantes.

\begin{abstract}
A gente faz a monitoração hemodinâmica desse paciente, avalia a perfusão tecidual, a ventilação, ausculta pulmonar, cardíaca, monitoração dos sinais vitais constantemente e toma as condutas adequadas caso não estejam estáveis. Enfim, avaliamos constantemente a clínica dele, para que esse paciente fique o mais estável possível para que os órgãos estejam viáveis para doação. (E10).
\end{abstract}

A terceira categoria, "Percebendo a dificuldade em compreender o processo de ME”, está atrelada às condições intervenientes. Revela que alguns profissionais, despreparados quanto às especificidades desse atendimento, ao cuidarem de pacientes no processo de ME, agem como se cuidassem de um paciente em óbito. Por esse motivo, não compreendem a necessidade de dar continuidade ao cuidado junto a esses pacientes.

Têm muitas pessoas que falam: "Ah, a gente está cuidan-
do de um paciente morto, então porque vai dar antibió-
tico para o paciente que está morto?" [...] Como eu falei,
tem uma mentalidade um pouco atrasada, assim de fa-
lar: "To cuidando de um paciente morto". (E1).

Assim como alguns profissionais que lidam diretamente com os pacientes nessas condições e não compreendem a ME, os familiares também têm dificuldade de compreender o diagnóstico, principalmente pela presença de batimentos cardíacos e movimentos respiratórios, e também o processo de doação de órgãos e tecidos para transplantes.

Acho que é dificil para a família entender [...] E eles perguntam: "Ah, mas o coração está batendo e não tem mais nada a ser feito?" É difícil aceitar a ME; aceitar que não tem mais vida ali. (E9). 
A quarta categoria, "Reconhecendo a importância da equipe multiprofissional no cuidado com o paciente no processo de ME", refere-se às estratégias. Revela que os enfermeiros possuem diversas atribuições técnicas, no que se refere à assistência direta aos pacientes em processo de ME, além de serem articuladores das relações interpessoais de todos os envolvidos nesse contexto e supervisor da equipe técnica de enfermagem durante a assistência prestada ao paciente em ME. Sua participação caracteriza-se principalmente pela presença contínua durante todo o processo em relação aos demais profissionais.

O enfermeiro é fundamental. Ele que vai mediar toda essa situação, tanto com a família quanto lidar com a questão do que precisa para o diagnóstico, quanto orientar e supervisionar a equipe de enfermagem. Ele vai estar sempre intermediando as situações. (E1).

O enfermeiro acompanha esse paciente 24 horas por dia sem ter nenhum tipo de ausência. Sempre vai ter um técnico ou um enfermeiro do lado daquele paciente, ao contrário de outros profissionais, fisioterapeuta, médico; eles não tão aqui o tempo todo. Apesar de eles estarem na UTI, eles não estão com o paciente o tempo todo. (E4).

Os dados também evidenciaram que a CIHDOTT é composta por uma equipe multiprofissional qualificada e com competências desenvolvidas para lidar com essa realidade. Consequentemente, faz a diferença no cuidado com o paciente em ME. Mostraram ainda que houve significativas mudanças após a implantação dessa comissão na instituição hospitalar.

\footnotetext{
Os profissionais que compõem essa equipe multiprofissional são extremamente preparados [...] Têm uma linguagem muito clara, competência, compromisso com o processo doação de órgãos. É fantástico! Eu penso que eles estão bem preparados [Equipe CIHDOTT] e estão sempre dispostos a dar informações. Acho que melhorou muito com a CIHDOTT, na parte de transplante de órgãos no estado. (D3).
}

A quinta categoria, "Relacionando o cuidado com o paciente no processo de ME à possibilidade de salvar outras vidas", está atrelada ao componente consequência. Destaca que a assistência de enfermagem prestada aos pacientes com diagnóstico de ME, de forma intensiva e contínua, é destinada à manutenção do organismo para possibilitar a viabilização dos órgãos e tecidos para doação e consequente transplante.
Eu cuido desse paciente de forma intensiva, para que ocorra a doação de órgãos. Todo esse controle, todo esse rigor, toda essa criteriosidade tem a finalidade de doação de órgãos. Só existe esse cuidado constante e intenso, pois existe a possibilidade de doação de órgãos. Caso contrário, cuidaríamos, mas não da forma intensa em que é realizada a assistência. Afinal, ele estaria em óbito. Cuidariamos com toda qualidade e respeito, mas não com tanta intensidade. (E6).

Entretanto, o desfecho da doação de órgãos é dado pelos familiares que possuem o poder de decisão sobre o processo. Nesse sentido, todos os profissionais envolvidos direta e indiretamente no cuidado com o paciente no processo de ME são responsáveis pelo cuidado e atendimento adequados aos familiares dos possíveis doadores, visando gerar uma resposta positiva à doação de órgãos e tecidos.

Acho que todos têm participação na sensibilização, para fazer com que a família posteriormente possa pensar em doar o órgão dessa pessoa que está aqui dentro. (E5).

Os dados revelaram também que cuidar de um paciente em ME é reconhecer, no processo de doação, a possibilidade de mudar a vida de outras pessoas que estão na fila de transplante de órgãos e tecidos. O cuidado adequado com o paciente e com os familiares desses pacientes gera a oportunidade de uma nova vida para outras pessoas.

Cuidar desse paciente em ME, é oportunizar a família a fazer uma boa ação para outras pessoas, disponibilizando os órgãos para que outras pessoas possam viver mais, ou numa condição melhor de vida [...] E significa, principalmente, ver outras pessoas vivendo melhor, numa condição melhor, com o órgão recebido através de uma doação. (C1).

Quando tu cuidas de um paciente em ME tu vais dar viabilidade a várias pessoas. Ofereces a chance de melhorar a vida de outras pessoas, se tu cuidar adequadamente daquele individuo. (E10).

\section{Discussão}

Os achados deste estudo apontam a UTI como o ambiente adequado para realizar os cuidados aos pacientes no processo de ME. Este achado corrobora a literatura, que confirma a ME como um diagnóstico que provém de quadros clínicos potencialmente graves que, em grande maioria, são tratados em UTIs, setores dotados de monitorização contínua a pacientes graves ou 
com descompensação de um ou mais sistemas orgânicos. Devido às características peculiares da UTI e à presença constante de uma equipe multiprofissional qualificada para o atendimento dos pacientes, é que o cuidado realizado com os pacientes no processo de ME nessa unidade é seguro e efetivo. Para atender à finalidade de manter a estabilidade orgânica, oferta suporte cardiocirculatório, ventilatório, endócrino, metabólico e hemodinâmico ${ }^{(14-15)}$, possibilitando o resultado final de todo o processo. Em contrapartida, em algumas realidades, tanto no Brasil quanto em outros países do mundo, como o Irã, o cuidado no processo de ME dá-se nas emergências, ambientes que, por suas características, também são dotados de aparatos tecnológicos e profissionais aptos para lidar com essa realidade ${ }^{(6)}$.

Estudos nacionais e internacionais esclarecem que cuidar de pacientes no processo de ME requer cuidados de múltiplos profissionais altamente qualificados para atuarem contínua e conjuntamente de forma intensiva e rigorosa na preservação dos órgãos e tecidos para transplantes ${ }^{(6,16)}$. Os enfermeiros, sobretudo os intensivistas, frente a essa realidade, precisam estar capacitados para identificar as alterações fisiopatológicas, para que, junto à equipe multiprofissional, possam instituir medidas terapêuticas adequadas para o cuidado com esses pacientes ${ }^{(7)}$.

Ainda que os pacientes em ME não apresentem possibilidades terapêuticas de cura em relação aos demais pacientes, é necessário que a assistência seja prestada com qualidade, segurança e respeito. Esses procedimentos visam não somente possibilitar a doação de órgãos e tecidos e efetivar o tratamento de múltiplos receptores, mas principalmente porque o corpo apresenta uma série de significados para familiares, profissionais e receptores dos órgãos e tecidos doados ${ }^{(17-18)}$.

Ressalta-se que, na UTI, os profissionais de saúde adotam, com o tempo, um mecanismo de adaptação ao trabalho e, muitas vezes, não se sensibilizam com o sofrimento dos familiares e a condição de saúde/doença dos pacientes. É comum visualizar um cuidado realizado pelos profissionais de saúde de modo rotineiro, mecanizado e técnico, priorizando a dimensão biológica em detrimento das demais dimensões do ser humano ${ }^{(13)}$.

Este estudo não identificou de forma explícita um cuidado mecanicista centrado na fisiopatologia do paciente. Confirma, entretanto, que é primordial que os enfermeiros e demais profissionais de saúde valorizem a assistência integral nesse ambiente de cuidado, reconhecendo os aspectos biopsicossociais de cada indivíduo, como parte do processo de humanização da assistência em UTI e na ME e de melhoria da qualidade da assistência. Corrobora ainda que a equipe de saúde lida não somente com o corpo físico, mas também com as demais dimensões desse indivíduo, da família e da sociedade ${ }^{(13)}$.

É nesse complexo cenário que os enfermeiros desempenham significativo papel no cuidado com os pacientes em ME. Estudo desenvolvido no Japão destacou que a participação dos enfermeiros, devido aos conhecimentos e olhares amplos que possuem diante das demandas de saúde da população, e sua participação nos processos de ME, têm contribuído para os avanços no número de doações de órgãos e tecidos para transplantes $^{(14)}$.

Em contrapartida, ainda que haja um significativo avanço na ciência, um aparato cada vez maior de tecnologia e de profissionais qualificados para lidar com a saúde e com a ME especificamente, ainda é muito comum que os profissionais de saúde não compreendam esse processo. Entretanto, esta é uma realidade florescente no Brasil e no mundo e influencia diretamente na qualidade do cuidado prestado por esses profissionais ${ }^{(6)}$.

Estudo desenvolvido em países asiáticos afirma que a relutância identificada no sistema de saúde, por questões relacionadas à religião, à cultura e à ética, inclusive entre os próprios profissionais de saúde, motivam a estagnação verificada na taxa de doação de órgãos e tecidos ${ }^{(18)}$. Estudo espanhol destaca que os profissionais de saúde, especialmente os enfermeiros, têm conhecimento sobre a ME, entretanto há uma 
desconfiança em relação à exatidão diagnóstica e efetividade dos protocolos de $\mathrm{ME}^{(16)}$.

Desse modo, o conhecimento dos profissionais de saúde acerca do diagnóstico de ME e demais cuidados que envolvam esse processo ainda é insuficiente, necessitando de formação específica, por meio da educação continuada, para ampliar a qualidade do cuidado prestado, refletindo, consequentemente, no aumento da oferta de órgãos e tecidos para transplantes no mundo e no Brasil ${ }^{(6,18-19)}$.

Ainda no que tange à educação, destaca-se a necessidade de inclusão consistente da temática no processo de formação, para favorecer o lidar com a ME de forma segura, qualificada e efetiva. Estudo desenvolvido com graduandos da saúde de uma instituição de ensino superior revelou o pouco e frágil conhecimento que possuem acerca desse tema e seus aspectos, além dos cuidados com os possíveis doadores. Associado a este fato estão os frágeis currículos de graduação, que abordam pouco essa temática, o que é alarmante, uma vez que os futuros profissionais saem da academia despreparados para atuar com essa realidade ${ }^{(20-21)}$. A falta de informação e de conhecimento acerca da ME, também faz com que os familiares, nesse momento de tristeza e luto, mantenham a esperança na recuperação do quadro clínico do paciente, principalmente por desconhecer a fisiopatologia da ME. Características como o corpo aquecido e o coração em atividade, dificultam, de fato, a compreensão da $\mathrm{ME}^{(22)}$.

Os enfermeiros, considerando essa possibilidade, devem assumir o papel de educadores, com a finalidade de oportunizar a melhora no relacionamento entre paciente, família e equipe, assim como na compreensão do processo em sua totalidade. Para tanto, é preciso despertar o encorajamento na aquisição de conhecimento, que conduza o profissional de saúde e os familiares ao autoconhecimento ${ }^{(22-23)}$. A atuação dos enfermeiros como educadores não deve estar atrelada e limitada aos espaços das UTIs ou demais espaços hospitalares, mas ampliada aos diversos espaços públicos e sociais, como forma de aumentar a conscientização da população a respeito do tema ${ }^{(21)}$.

Além disso, estar com a família do potencial doador é também uma atribuição do enfermeiro assistencial e/ou enfermeiro da CIHDOTT e demais profissionais da equipe de saúde. Entretanto, este é um cenário de conflito vivenciado por esses profissionais, sendo o momento mais difícil e estressante de todo o processo. Com isso, os enfermeiros devem estar capacitados, pois os familiares estão enfrentando um momento difícil e doloroso, sendo de suma importância contar com o apoio dos profissionais, para thes proporcionar conforto e segurança e ajudá-los a compreender melhor a ME, aumentando as chances do consentimento para o processo de doação de órgãos ${ }^{(3,7,15)}$.

Estudos nacionais e internacionais apontam que os enfermeiros devem proporcionar uma explicação com terminologias claras e consistentes, considerando a realidade sociocultural e religiosa dos familiares, para assegurar-lhes a compreensão, antes de discutir o processo de doação de órgãos e tecidos para transplantes. Com esse cuidado, evitarão futuros desentendimentos por parte dos familiares ${ }^{(7,13,22-23)}$.

O Conselho Federal de Enfermagem (COFEN) também atribui ao enfermeiro o planejamento, execução, articulação, coordenação, supervisão e avaliação dos procedimentos de enfermagem prestados aos possíveis doadores de órgãos e tecidos. Salienta-se que, além das suas atribuições, os enfermeiros e respectivas equipes são essenciais nessa realidade, uma vez que estarão assistindo esses pacientes 24 horas, melhorando a qualidade do cuidado prestado e ampliando a potencialidade dos órgãos e tecidos para o processo de doação e transplantes ${ }^{(5,10)}$.

Destaca-se que o cuidado com o paciente no processo de ME é configurado como multiprofissional, tendo em vista que o enfermeiro e a equipe de enfermagem trabalham em conjunto com os demais profissionais, ampliando a qualidade e efetividade da assistência prestada. Afinal, nenhum profissional é capaz de individualmente responder pelas diferentes dimensões 
que envolvem o cuidado com o paciente no processo de $\mathrm{ME}^{(5,8,11)}$.

Os profissionais da CIHDOTT envolvidos nesse processo têm atribuições de educação permanente da equipe multiprofissional sobre os aspectos de doação e transplante de órgãos e tecidos, habilidade de comunicação interpessoal, conhecimento das legislações específicas, aspectos éticos e documentos para a doação de órgãos e tecidos, promoção da integração com as unidades que dispõem de recursos diagnósticos, além de organizar rotinas e protocolos que possibilitem o processo de doação e captação de órgãos e tecidos ${ }^{(15)}$. O envolvimento desses profissionais tem permitido melhor organização e identificação dos potenciais doadores, abordagem mais adequada dos familiares e maiores articulações dos hospitais com as centrais de notificação, captação e doação de órgãos, ampliando qualitativa e quantitativamente esse processo $^{(5,15)}$.

Este estudo apresenta como limitações o fato de ser desenvolvido em um único cenário, não permitindo generalizar seus resultados. Entretanto, sua importância reside na possibilidade de subsidiar a reflexão e a prática de enfermeiros e demais profissionais de saúde em cenários similares.

\section{Conclusão}

O presente estudo revelou que o cuidado prestado aos pacientes em processo de ME é significado pelos enfermeiros intensivistas como gerador da oportunidade de uma nova vida aos múltiplos receptores. Significa propiciar, por meio da doação de órgãos e tecidos, a oportunidade de salvar vidas de pessoas para as quais a única possibilidade terapêutica, para uma vida de qualidade e produtividade, é o produto final de todo o processo: o transplante. É por meio do acordo realizado com os familiares pela equipe multiprofissional assistencial e principalmente da CIHDOTT, que a doação de órgãos e tecidos efetiva-se, possibilitando a cura de uma série de pacientes que aguardam nas filas de transplantes de órgãos e tecidos por todo o país.
Embora o estudo tenha sido desenvolvido em uma única realidade e não tenha a potencialidade de generalização, espera-se que desperte nos enfermeiros que atuam junto à equipe multiprofissional, a necessidade de assumir novas posturas diante desse cenário, principalmente de educador em saúde tanto para pacientes quanto principalmente para familiares e sociedade, como forma de potencializar a aceitação da doação de órgãos e tecidos para transplantes.

Assim, sugere-se o desenvolvimento de mais estudos acerca da ME e em outras realidades, que possam estimular ainda mais a construção, discussão e reflexão dos estigmas acerca da ME entre os enfermeiros e demais profissionais de saúde, com vistas a desenvolver a qualidade da assistência com os pacientes e familiares no processo de ME, na doação de órgãos e tecidos para transplantes e possíveis receptores.

\section{Colaborações:}

1. concepção, projeto, análise e interpretação dos dados: Murilo Pedroso Alves, Franciele da Silva Rodrigues, Giovana Dorneles Callegaro Higashi, Eliane Regina Pereira do Nascimento e Alacoque Lorenzini Erdmann;

2. redação do artigo e revisão crítica relevante do conteúdo intelectual: Murilo Pedroso Alves, Franciele da Silva Rodrigues, Kamylla Santos da Cunha, Giovana Dorneles Callegaro Higashi, Eliane Regina Pereira do Nascimento e Alacoque Lorenzini Erdmann;

3. aprovação final da versão a ser publicada: Murilo Pedroso Alves, Kamylla Santos da Cunha, Giovana Dorneles Callegaro Higashi, Eliane Regina Pereira do Nascimento e Alacoque Lorenzini Erdmann.

\section{Referências}

1. Associação Brasileira de Transplante de Órgãos. 2017 - Dimensionamento dos Transplantes no Brasil e em cada estado (2010-2017). Registro Brasileiro de Transplantes: Veículo Oficial da Associação Brasileira de Transplante de Órgãos [Internet]. São Paulo; 2017 [cited 2018 Aug 22];ano XXIII(4). Available from: http://www.abto.org. 
br/abtov03/Upload/file/RBT/2017/rbt-imprensaleitura-compressed.pdf

2. Conselho Federal de Medicina. Resolução CFM n. 1.480 , de 8 de agosto de 1997. Estabelece os critérios para caracterização de morte encefálica. Brasília; 1997 [cited 2018 Aug 22]. Available from: http://www.portalmedico.org.br/resolucoes/ cfm/1997/1480_1997.htm

3. MoraesEL, SantosMJ,MerighiMAB, MassarolloMCKB. Vivência de enfermeiros no processo de doação de órgãos e tecidos para transplante. Rev Latino-Am Enfermagem. 2014 [cited 2018 Nov 29];22(2):22633. Available from: http://www.scielo.br/pdf/rlae/ v22n2/pt_0104-1169-rlae-22-02-00226.pdf

4. Passos IMS, Figueiredo JBV, Menezes MO, Silva DP, Oliveira DML. Manutenção hemodinâmica na morte encefálica: revisão literária. Cad Grad Ciênc Hum Soc Unit [Internet]. 2014 [cited 2018 Nov 30];2(1):73-86. Available from: https:// periodicos.set.edu.br/index.php/ cadernobiologicas/article/view/1339

5. Cavalcante LP, Ramos IC, Araújo MA, Alves MD, Braga VA. Cuidados de enfermagem ao paciente em morte encefálica e potencial doador de órgãos. Acta paul enferm [Internet]. 2014 [cited 2018 Oct 12];27(6):567-72. Available from: http://www.scielo. br/pdf/ape/v27n6/1982-0194-ape-027-006-0567. pdf

6. Masoumian Hoseini ST, Manzari Z, Khaleghi I. ICU Nurses' Knowledge, Attitude, and Practice Towards their Role in the Organ Donation Process from Brain-Dead Patients and Factors Influencing it in Iran. Int J Organ Transplant Med [Internet]. 2015 [cited 2018 Nov 16];6(3):105-13. Available from: https://www.ncbi.nlm.nih.gov/pubmed/26306156

7. Doria DL, Leite PMG, Brito FPGB, Brito GMG, Resende GGS, Leite FL, et al. Conhecimento do enfermeiro no processo de doação de órgãos. Enferm foco [Internet]. 2015 [cited 2019 Feb 20];6(1/4):31-5. Available from: http://revista. cofen.gov.br/index.php/enfermagem/article/ viewFile/573/255

8. Youn TS, Greer DM. Brain Death and Management of a Potential Organ Donor in the Intensive Care Unit. Crit Care Clin [Internet]. 2014 [cited 2018 Oct 12];30(4):813-31. Available from: https://www. ncbi.nlm.nih.gov/pubmed/25257743

9. Associação Brasileira de Transplante de Órgãos. 2015 - Dimensionamento dos Transplantes no Brasil e em cada estado (2008-2015). Registro
Brasileiro de Transplantes: Veículo Oficial da Associação Brasileira de Transplante de Órgãos [Internet]. São Paulo; 2015 [cited 2018 Aug 22]; ano XXI(4). Available from: http://www.abto.org.br/ abtov03/Upload/file/RBT/2015/anual-n-associado. pdf

10. Ministério da Saúde. Conselho Federal de Enfermagem. Resolução COFEN n. 292, de 7 de junho de 2004. Normatiza a atuação do Enfermeiro na Captação e Transplante de Órgãos e de Tecidos. Rio de Janeiro; 2004 [Internet]. [cited 2018 Aug 22]. Available from: http://www.cofen.gov.br/resoluocofen-2922004_4328.html

11. Strauss A, Corbin J. Pesquisa qualitativa: técnicas e procedimentos para o desenvolvimento de teoria fundamentada. Tradução Luciane de Oliveira da Rocha. 2a ed. Porto Alegre: Artmed; 2008.

12. Brasil. Ministério da Saúde. Conselho Nacional de Saúde. Resolução no 466, de 12 de dezembro de 2012. Aprova diretrizes e normas reguladoras de pesquisas envolvendo seres humanos. Brasília; 2012 [Internet]. [cited 2018 Aug 22]. Available from: http://bvsms.saude.gov.br/bvs/saudelegis/ cns/2013/res0466_12_12_2012.html

13. Jakimowicz S, Perry L. A concept analysis of patientcentred nursing in the intensive care unit. J adv nurs [Internet]. 2015 [cited 2018 Sep 24];71(7):1499-517. Available from: https://onlinelibrary.wiley.com/ doi/full/10.1111/jan.12644

14. Soyama A, Eguchi S. The current status and future perspectives of organ donation in Japan: learning from the systems in other countries. Surg today [Internet]. 2015 [cited 2018 Sep 24];46(4):387-92. Available from: https://link.springer.com/article/ 10.1007/s00595-015-1211-6

15. Freire ILS, Vasconcelos QLDAQ, Melo GSM, Torres GV, Araújo EC, Miranda FAN. Facilitating aspects and barriers in the effectiveness of donation of organs and tissues. Texto contexto enferm [Internet]. 2014 [cited 2018 Sep 24];23(4):925-34. Available from: http://www.scielo. br/scielo.php?script=sci_arttext\&pid= S0104-07072014000400925

16. Lomero MM, Jiménez-Herrera MF, Rasero MJ, Sandiumenge A. Nurses' attitudes and knowledge regarding organ and tissue donation and transplantation in a provincial hospital: A descriptive and multivariate analysis. Nurs health sci [Internet]. 2017 [cited 2018 Oct 12];19(3):32230. Available from: https://www.ncbi.nlm.nih.gov/ pubmed/28631279 
17. Cavalcante LP, Ramos IC, Araújo MAM, Alves MDS, Braga VAB. Cuidados de enfermagem ao paciente em morte encefálica e potencial doador de órgãos. Acta Paul Enferm [Internet]. 2014 [cited 2018 Oct 12];27(6):567-72. Availabe from: https://www. redalyc.org/html/3070/307032877012/

18. Chua HC, Kwek TK, Morihara H, Gao D. Brain Death: The Asian Perspective. Semin Neurol [Internet]. 2015 [cited 2018 Sep 24];35(2):152-61. Available from: https://www.thieme-connect.com/products/ ejournals/html/10.1055/s-0035-1547539\#N69678

19. Vesco NL, Nogueira CS, Lima RF, Souza VN, Brasil BMBL, Viana CDMR. Conhecimento do enfermeiro na manutenção do potencial doador de órgãos e tecidos para transplante. Rev enferm UFPE on line. 2016 [cited 2018 Sep 24];10(5):1615-24. Available from: https://periodicos.ufpe.br/revistas/ revistaenfermagem/article/viewFile/11157/12675

20. Soares LMD, Leite RG, Rocha FCV. Conhecimento dos graduandos de uma instituição de ensino superior sobre a doação de órgãos. R Interd [Internet]. 2015 [cited 2018 Oct 12];8(2):158-68. Available from: https://revistainterdisciplinar. uninovafapi.edu.br/index.php/revinter/article/ view/505/pdf_218
21. Pauletto MR, Beuter M, Timm AMB, Santos NO, Maldaner CR, Bruisma JL. Motivos para pacientes em hemodiálise não ingressarem em lista de transplante. Rev baiana enferm [Internet]. 2016 [cited 2018 Oct 16];30(3):1-11. Available from: https://portalseer.ufba.br/index.php/enfermagem/ article/view/15987

22. Moraes EL, Neves FF, Santos MJ, Merighi MAB, Massarollo MCKB. Experiências e expectativas de enfermeiros no cuidado ao doador de órgãos e à sua família. Rev Esc Enferm USP [Internet]. 2015 [cited 2018 Oct 18];49(Esp2):129-35. Available from: http://www.periodicos.usp.br/reeusp/article/ view/112655/110552

23. Castro MFS, Rocha RLP, Fialho LP, Silva PAT, Oliveira RSP, Costa ML. Conhecimento e atitude dos enfermeiros frente ao processo de doação de órgãos. Rev Med Minas Gerais [Internet]. 2018 [cited 2018 Oct 18];28(Supl 5):e-S280504. Available from: http://rmmg.org/artigo/detalhes/2436

Recebido: 5 de dezembro de 2018

Aprovado: 26 de fevereiro de 2019

Publicado: 18 de junho de 2019

A Revista Baiana de Enfermagem utiliza a Licença Creative Commons - Atribuição-NãoComercial 4.0 Internacional. https://creativecommons.org/licenses/by-nc/4.0/ Este artigo é de acesso aberto distribuído sob os termos da Licença Creative Commons (CC BY-NC). Esta licença permite que outros remixem, adaptem e criem a partir do seu trabalho para fins não comerciais. Embora os novos trabalhos tenham de lhe atribuir o devido crédito e não possam ser usados para fins comerciais, os usuários não têm de licenciar esses trabalhos derivados sob os mesmos termos. 